\title{
Smart Home System Based on ZigBee Network and STM32F407 Micro- processor
}

\author{
Yongliang Zhang ${ }^{1, *}$ and Ling $\mathrm{Li}^{2}$ \\ ${ }^{I}$ College of Information Engineering, Guangdong Mechanical and Electrical College, Guangzhou, Guangdong, 510550, \\ China \\ ${ }^{2}$ Shenzhen Institute of Advanced Technology, Chinese Academy of Sciences, Shenzhen, Guangdong, 518055, China
}

\begin{abstract}
This paper proposed a new smart home system that is designed based on ZigBee network and STM32F407 microprocessor. The system architecture, the basic circuit diagram and the flowchart of the software system are also provided. This smart home system integrates home appliances, lighting system, entry guard system, smoke sensors, automatic curtains and other equipment by connecting them to a ZigBee network. An STM32F407 MCU is used as the main control microprocessor to implement the interconnection among ZigBee, GPRS and WiFi networks. Users can use the smart home system by operations on a touch screen connecting STM32F407. By using a smart phone or tablet connected to a WiFi or GPRS network, users can also remotely monitor and control all home appliances in the smart home system.
\end{abstract}

Keywords: ZigBee Network, STM32F407 Microprocessor, Smart Home System.

\section{INTRODUCTION}

With the rapid development of information technologies, nowadays people have more requirements for safety, comfort, efficiency and transparency of their home environment. In every modern home, there are all kinds of household appliances, from the so-called intelligent refrigerator, air conditioner to traditional electric light and TV set, all of which generally suffer disadvantages of low flexibility, poor compatibility, expensive upgrade and maintenance cost. Moreover, all the electrical appliances work independently from one another instead of collaborating as an organic, organizational whole. House owners have no choice but to deal with these disorganized home appliances which consumes a lot of time, energy and money from day to day. Therefore, busy office workers have been longing for a smart home system featuring comprehensive functions, simple operation and convenient service. The concept of smart home was first proposed years, and the market demand has always existed. But due to the constraints of key technical breakthroughs, smart home systems have largely remained in the lab. With the emergence of Internet of Things technologies, especially the development of radio frequency technologies such as ZigBee, Bluetooth, $\mathrm{WiFi}$, it has become a hot research topic to design a household management system which can achieve seamless sensing and unified control by using the wireless connections of home appliances [1-3].

At present, there are two types of smart home technologies in the market, namely, the wired and the wireless. In a wired system, all control signals are transmitted by cables. But wired is a complicated task involving heavy workload, high cost, difficult maintenance, complex networking and poor expansibility. An ideal wireless smart home system needs to meet requirements of low power consumption, stable operation, and easy networking. The transmission speed is obviously not a key part for this type of applications. Therefore, compared to Bluetooth, $\mathrm{WiFi}, 315 \mathrm{M} / 433 \mathrm{M}$ radio frequency technology, the newly emerging Zibgee, as a short-range wireless network technology featuring low complexity, low power consumption, low rate, low cost, ad-hoc network, high reliability, high security, has gradually been applied to such fields as automatic control, remote control and intelligent household [4-6]. The system proposed in this paper uses the ZigBee network technology to build a home network to organically combine the household appliances, lighting system, security system, entry guard system, smoke alarm, automatic curtains. An STM32F407 microprocessor with Cortex M4 kernel is used to implement real-time monitoring for the smart home system. At the same time, with the gateway function, the smart system is able to provide interconnection among ZigBee, GPRS and WiFi networks and realize the internal network (WiFi) and the outside network (GPRS). Users can remotely operate the smart home system using a smart phone or tablet.

\section{SYSTEM ARCHITECTURE}

The smart home system mainly includes the smart lighting system, home security system, entry guard systems, home appliance systems, multi-functional sockets, smart electricity meters, wireless dimming lights, electric curtains, smoke alarm, multimedia systems, IP camera monitoring system. The overall structure is shown in Fig. (1). 


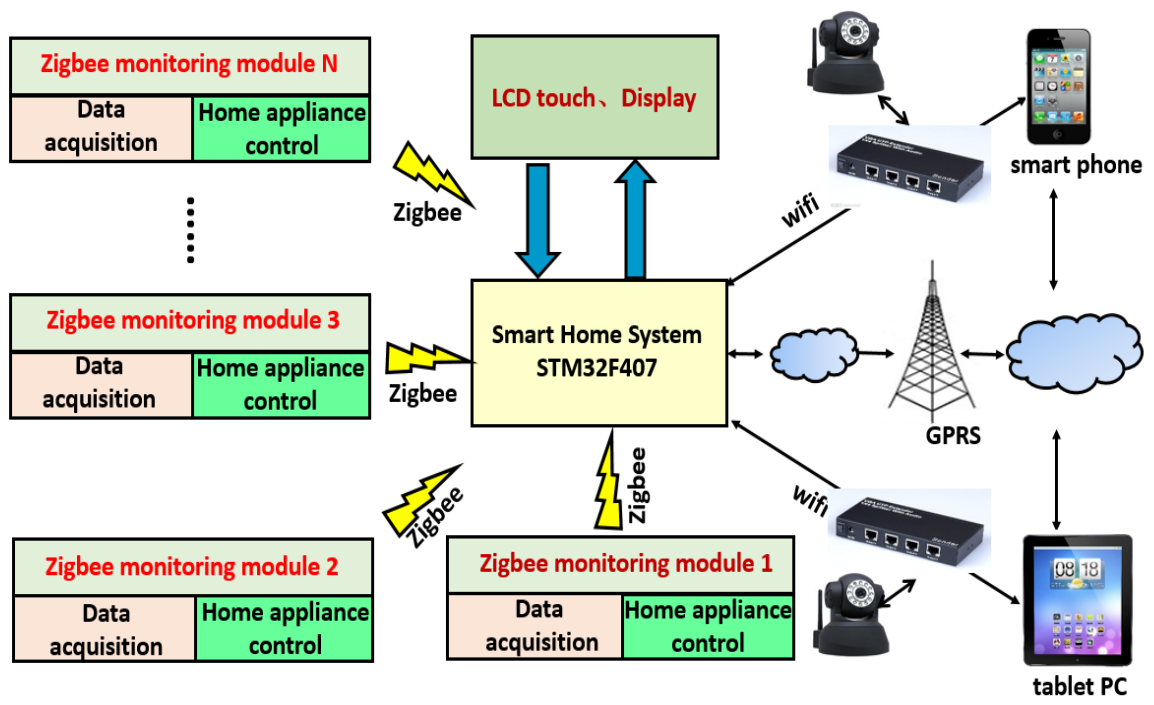

Fig. (1). Overall structure of the smart home system.

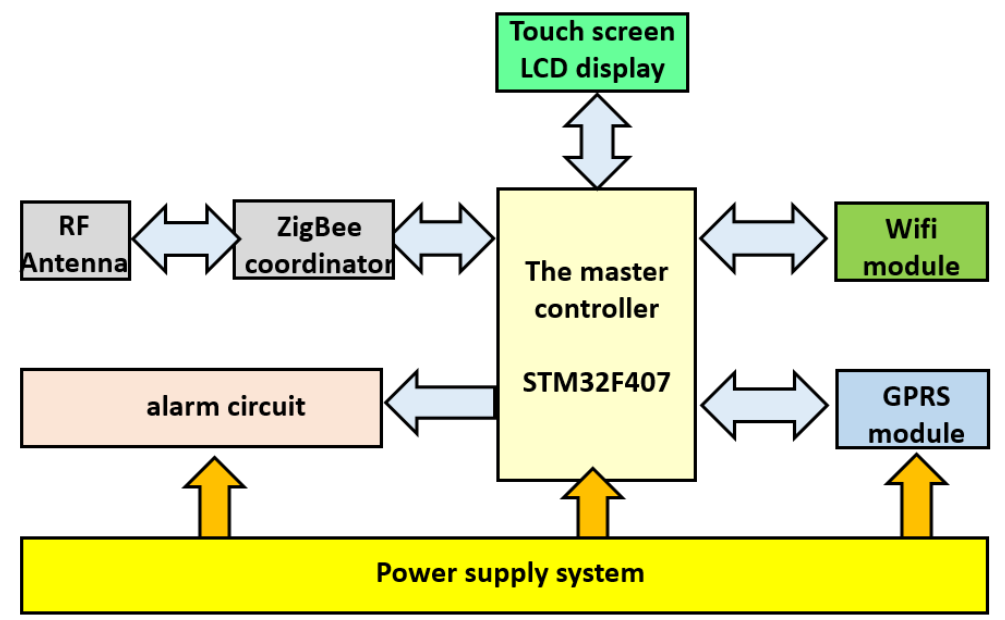

Fig. (2). Structure of the master controller.

Various sensors on home appliances are able to collect physical signals such as temperature, humidity, illumination, smoke density, and transmit the data to the ZigBee network nodes. In order to achieve the smart home system's intelligent control for TV sets, air conditioners, DVD and other home appliances, a infrared-ZigBee repeater need to be installed between the smart home system and the home appliances with infrared control features. ZigBee node signals from various lines, via ZigBee coordinator, are transmitted to the smart home system based on STM32 platform to implement the real-time monitoring of household system. With the gateway function gained by extending the GPRS and WiFi, the smart home system can connect the Zibgee network to $\mathrm{WiFi}$ and GPRS networks. In the home network (WiFi) mode, the smart home system is connected to the router as a WiFi client. Users can use the apps on smart phones or tablets on the same local area network to control home appliances. Away from home, users can remotely monitor the status of home appliances over the outside network (GPRS). In addition, multiple IP cameras are installed in the smart home system. In the WiFi network mode, users can view a variety of situations of home on their smart phones or tablet. Pyroelectric infrared capabilities can be expanded on IP cameras, with which IP cameras can coordinate with the security system. Once a moving object is detected in the monitored area, the system automatically starts recording videos, and notifies house owners.

\section{HARDWARE MODULE CIRCUIT DESIGN}

\subsection{Master Controller of the Smart Home System}

The smart home systems uses an STM32F407 series chip manufactured by STMicroelectronics [7, 8] as the MCU of its master control board. The chip is based on the highperformance architecture Cortex M4 32 bit RISC core designed by ARM, with an FPU unit, $1 \mathrm{MB}$ Flash, $192+4 \mathrm{~Kb}$ ram, working frequency up to $168 \mathrm{MHz}$. The master control board mainly includes the power supply module, main control chip, ZigBee coordinator, GPRS module, the WiFi module, LCD module, alarm module, etc. The hardware structure is shown in Fig. (2). 


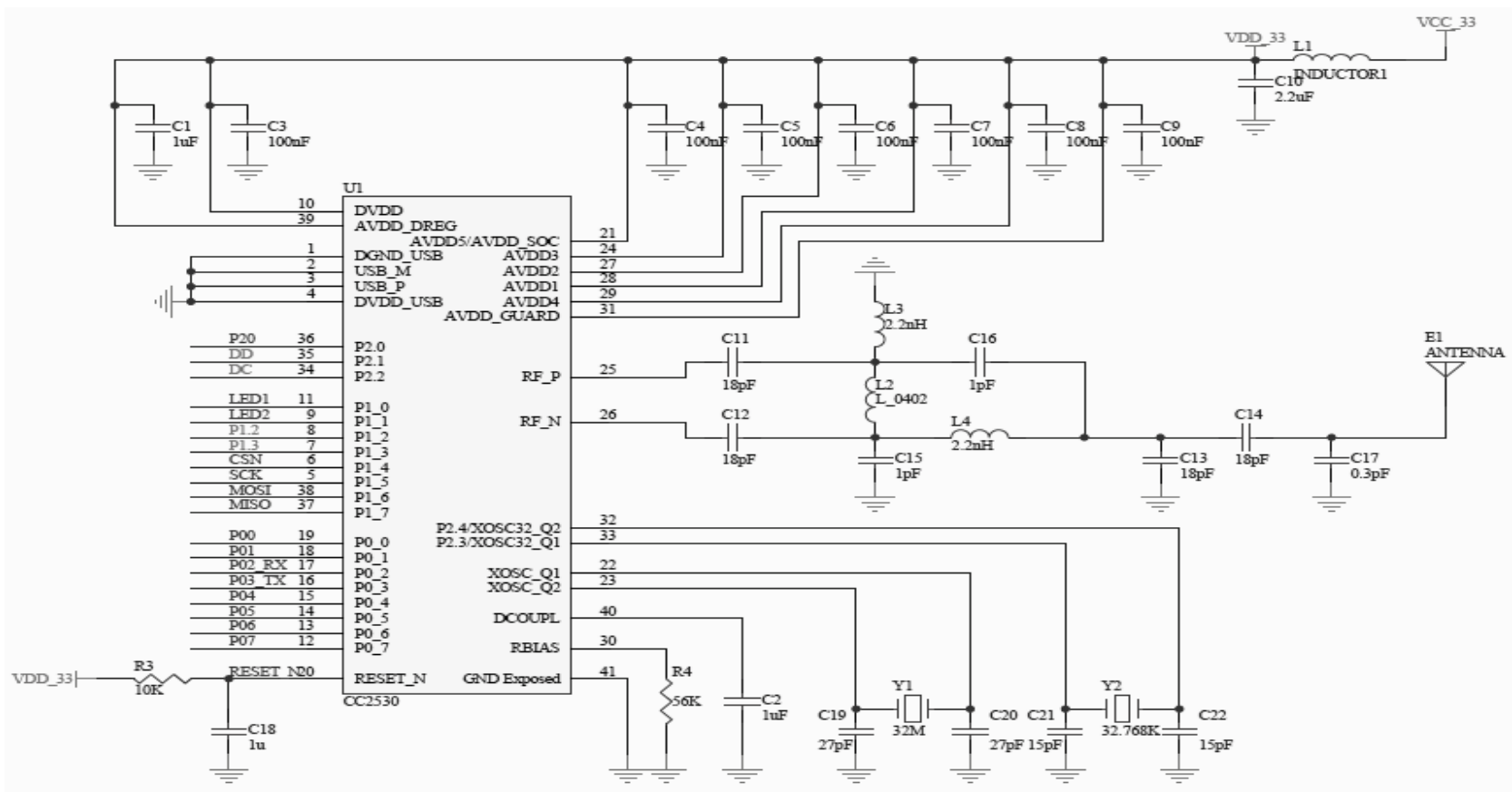

Fig. (3). CC2530 typical circuit.

RS232 serial ports are used between the main control chip, ZigBee coordinator and GPRS module for communication. The LCD module is a $800 * 480$-resolution color touch screen (model: AT070TN83 by Innolux). The touch and display functions are realized through the built-in FSMC controller of STM32F407. SIM900 is used as the GPRS module, a wireless communication module produced by SIMCom Company which is embedded with TCP/IP protocol stack. It supports GSM/GPRS communication and has an RF antenna and a local SIM card or the connection function. Controlled by AT commands, its RS232 serial port can easily realize serial communication with STM32F407. The WiFi module is a WM-09-G-MR with a 88 w8686 core and built-in IEEE802.11 wireless network protocol stack and TCP/IP protocol stack. The data between the main control board and wireless network is transmitted through the SDIO interface in STM32F407. A sound and light alarm circuit controlled by the I/O port of STM32F407 is used as the alarm module. A CC2530 chip produced by TI Company is used as the ZigBee coordinator. CC2530 is a real System-on-aProgrammable-Chip solution which is used in the $2.4 \mathrm{GHz}$ IEEE 802.15.4, ZigBee and RF4CE application. CC2530 integrates $2.4 \mathrm{GHz}$ high performance ZigBee RF front-end, memory, and micro controller on a single chip. Using an 8bit $8051 \mathrm{MCU}$, it has up to $256 \mathrm{~KB}$ programmable flash memory and $8 \mathrm{~KB}$ RAM and is also provided with the A/D converter, multi-general timer, AES128 coprocessor, watchdog timer, sleep-mode timer with $32 \mathrm{kHz}$ crystals, reset circuit, power detection circuit and 21 programmable $\mathrm{I} / \mathrm{O}$ pins.http://baike.baidu.com/view/1915042.htmhttp://baike.ba idu.com/view/882467.htm CC2530 typical circuit is shown in Fig. (3).

\subsection{ZigBee Network Nodes}

The ZigBee network node also adopts the CC2530 chip design. In the hardware platform of the network node, CC2530 mainly implements three functions: collecting ana$\log$ and digital information such as temperature and humidity, light intensity, smoke density, human body infrared radiation via $21 \mathrm{I} / \mathrm{O}$ ports and $8 \mathrm{~A} / \mathrm{D}$ converter of $12 \mathrm{bit}$; implementation of household appliances' infrared-ZigBee data forwarding, and control of sensing modules such as smart sockets, electric curtains; sending and receiving data between the wireless RF module and ZigBee coordinator.

\subsubsection{Acquisition of Temperature and Humidity, Light Intensity Information}

Light intensity and temperature humidity meters collect the temperatures, humidity and light of the sitting room and balcony, and then use the ZigBee network to for communication. A DHT11 digital temperature and humidity sensor is used for temperature and humidity data collection. The sensor includes a resistance-type moisture element and a NTC temperature measuring element that are connected to a highperformance 8-bit single chip microcomputer. This design has advantages of fast response, strong anti-interference ability and superior cost efficiency.http://baike.baidu.com/view/3457800.htm. The single-wire serial interface facilitates the connection with CC2530 IO port. Shown in Fig. (4) is DHT11 hardware circuit diagram. The smart home system uses a TSL2561 chip to detect the indoor light intensity. TSL2561 is a light intensity digital conversion chip produced by TAOS Company featuring high speed, low power 


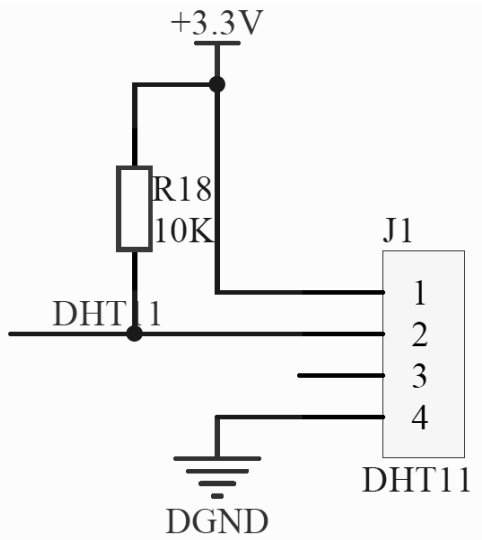

Fig. (4). DHT11 hardware circuit diagram.

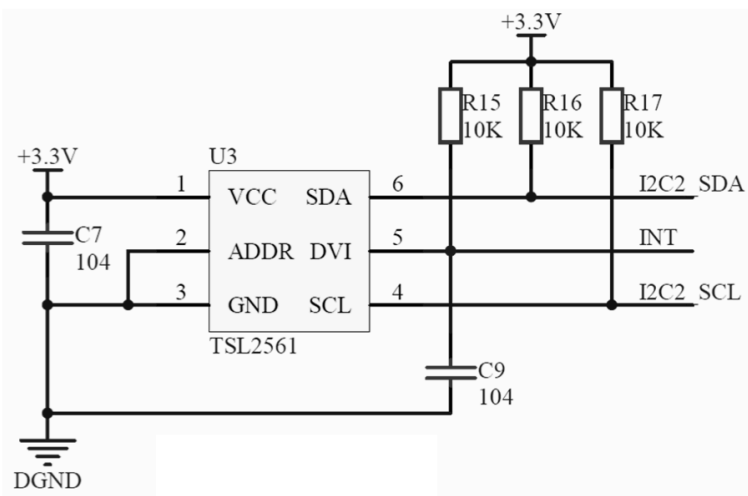

Fig. (5). Circuit diagram of light intensity sensor.

consumption, wide range, and programmability. It is connected to $\mathrm{CC} 2530$ by the $\mathrm{I} 2 \mathrm{C}$ bus. The typical circuit is shown in Fig. (5).

\subsubsection{Detection of Glass Breaking, Smoke Combustible Gas, Human Body Infrared Radiation}

The glass breaking detector adopts DS1101i model which is able to effectively detect the sound of glass breaking $(10 \mathrm{KHz} \sim 15 \mathrm{KHz})$ by using the piezoelectric effect of piezoelectric ceramic chip. The infrared detector adopts a YKQH811 hanging dual infrared detector. It can trigger the alarm when detecting invaders' approaching. The KW-SS168 photoelectric smoke detector can send an alarm with sound and light alarm in case of fire. The XSJ-888-X combustible gas detector, can sense the concentration of coal gas, natural gas, liquefied petroleum gas and other combustible gases. Output digital signals from the sensor modules are tested by CC2530 I/O and then transmitted to the master controller of the smart home system over the ZigBee network. Once the data exceeded a preset value, an alarm is triggered, which cooperates with the security system.

\subsubsection{Smart Socket}

The smart socket collects the real-time power supply and electricity information of home appliances such as TV set, air conditioners, electric cooker, and water heater. According to the information collected, the smart socket distributes power load for each appliance in an efficient manner. The circuit diagram is shown in Fig. (6-8).

Tested grid voltage, current signal respectively through voltage, current transformer, changed into detectable signals for electricity chip ADE7753, chip input terminal has the positive and negative input limiter protection diode, and achieve communication through the SPI bus interface and CC2530 network node, displayed in real-time measurement and power parameters, used for measuring the whole house of electricity, reasonable analysis the power load in the home.

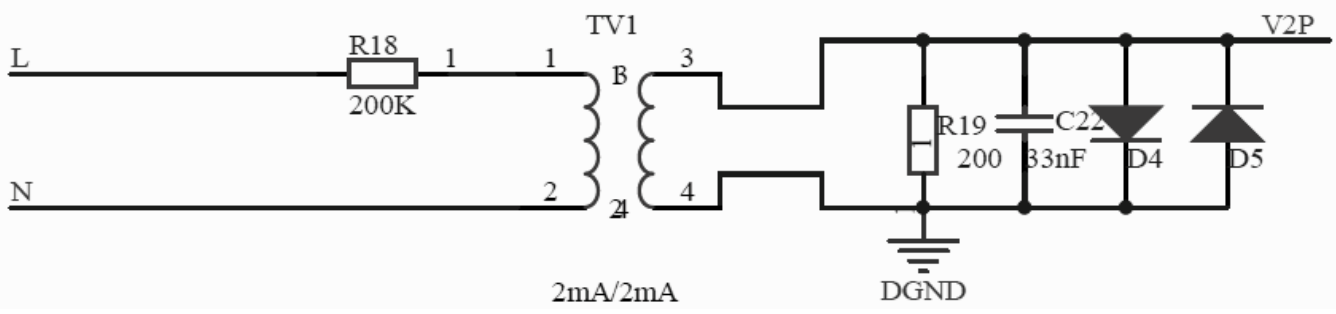

Fig. (6). Circuit diagram of voltage acquisition in the power network.

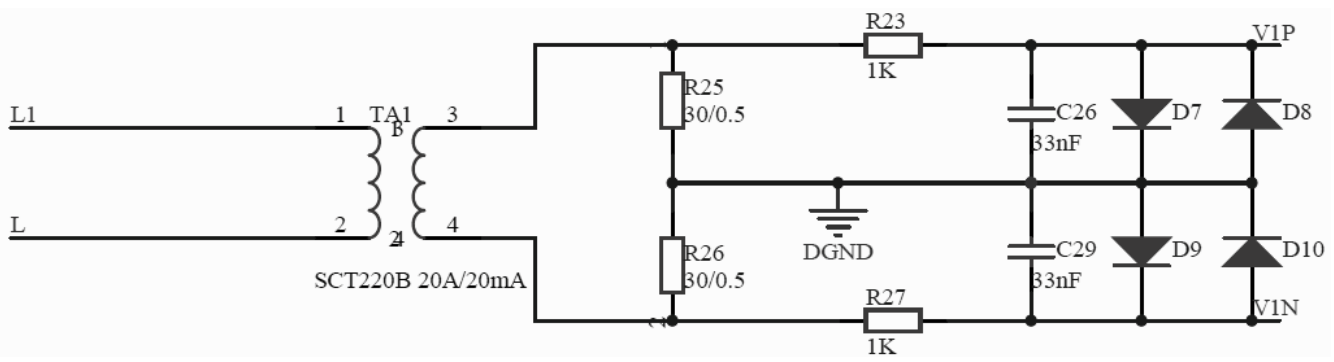

Fig. (7). Circuit diagram of the electric current acquisition in power network. 


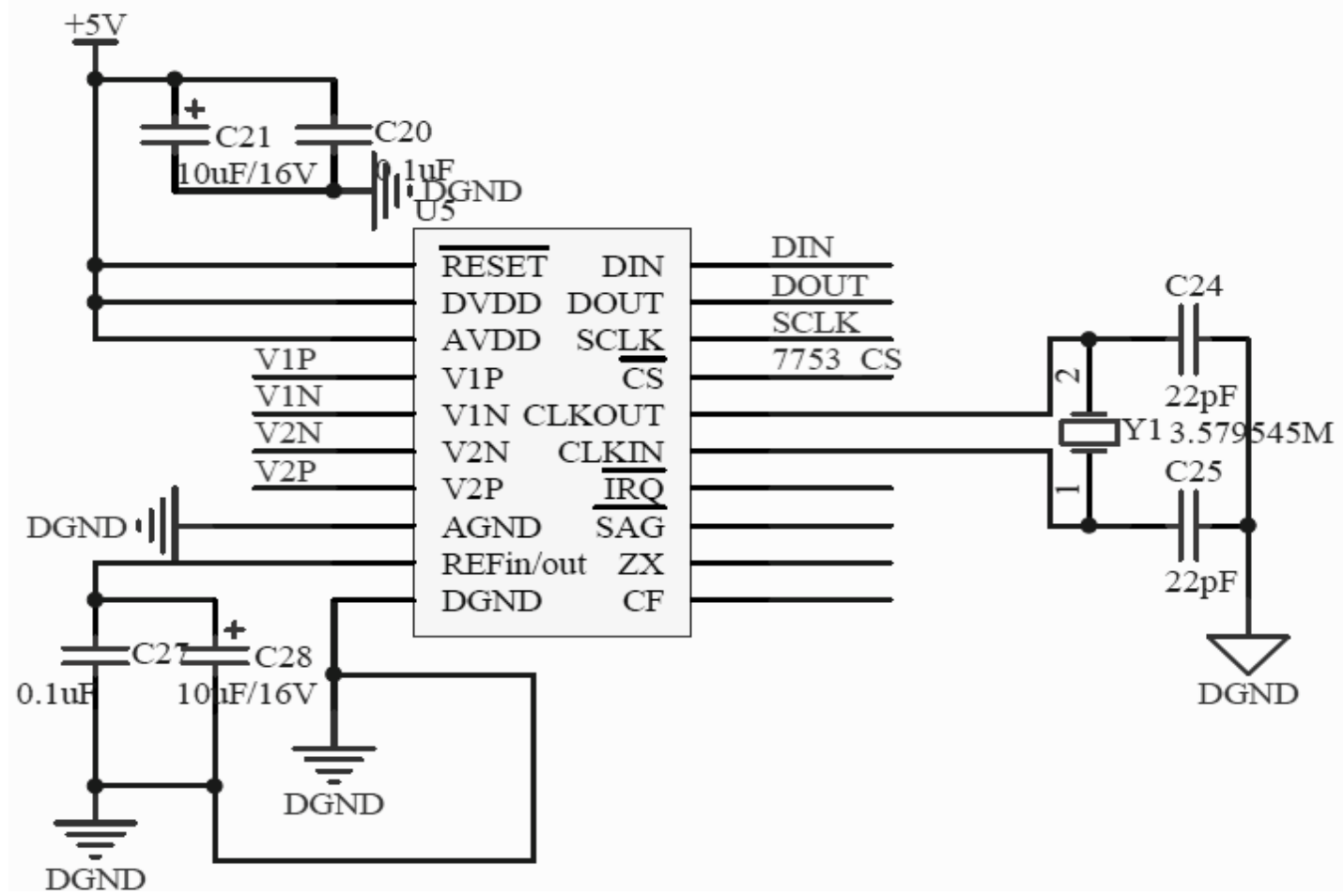

Fig. (8). Circuit diagram of ADE7753 electric parameter measurement.

\subsubsection{Infrared-ZigBee Forwarding Device}

The infrared-ZigBee forwarding device can learn, store, receive and forward infrared remote control signals of television, air conditioner and other home appliances. The device communicates through the RS232 serial port and the CC2530 network node. An HS0038 receiver is used for receiving infrared signals. Its working voltage $\mathrm{VCC}$ ranges from $3.7 \mathrm{~V}$ to $5.5 \mathrm{~V}$ with frequency $\mathrm{fc}=38 \mathrm{KHz}$. Its circuit diagram is shown in Fig. (9). A $0.3 \mathrm{w} / 940 \mathrm{~nm}$ infrared emitting diode is used for infrared emission with ULN2003 Darlington tube as its driver. The circuit diagram is shown in Fig. (10).

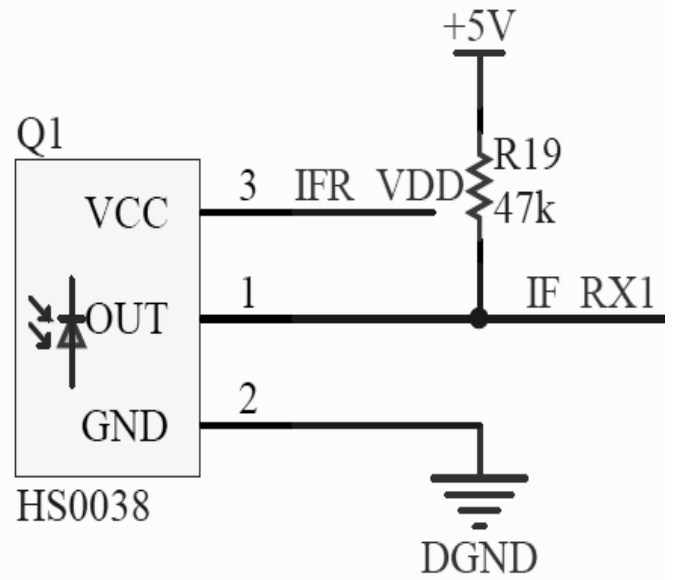

Fig. (9). Circuit diagram of infrared receiver.

\subsubsection{Electric Curtains \& Electric Windows}

The controller of electric curtains, electric window contains 4-way electric relay, as shown in Fig. (11). K1, K2 control curtain motors while $\mathrm{K} 3, \mathrm{~K} 4$ control window motors, respectively. The electric relay is driven by the ULN2003 via the CC2530 I/O port. ULN2003 sink current can be up to $500 \mathrm{~mA}$, and can withstand $50 \mathrm{~V}$ voltage in the OFF state. The output can be ensured even under high load current simultaneously.

\section{SYSTEM SOFTWARE DESIGN}

An STM32F407 microprocessor is used as the master controller of the smart home system. The ZigBee network node is designed with a CC2530 chip. System programs mainly include the main program, ZigBee coordinator program, ZigBee network node program, Android smart phone applications.

\subsection{Main Program Design of Smart Home System}

The main control microprocessor, as the core of the whole system, needs to perform multiple tasks. In order to coordinate the operation of each task, embedded operating system $\mu \mathrm{C} / \mathrm{OS}$-III is transplanted as the software platform [9]. A preemptive task scheduling mechanism based on task priority is used to classify the tasks on the main controller into ZigBee communication, touch screen response, LCD display, WiFi communication, GPRS communication, RS232 serial communication according to their priority and 


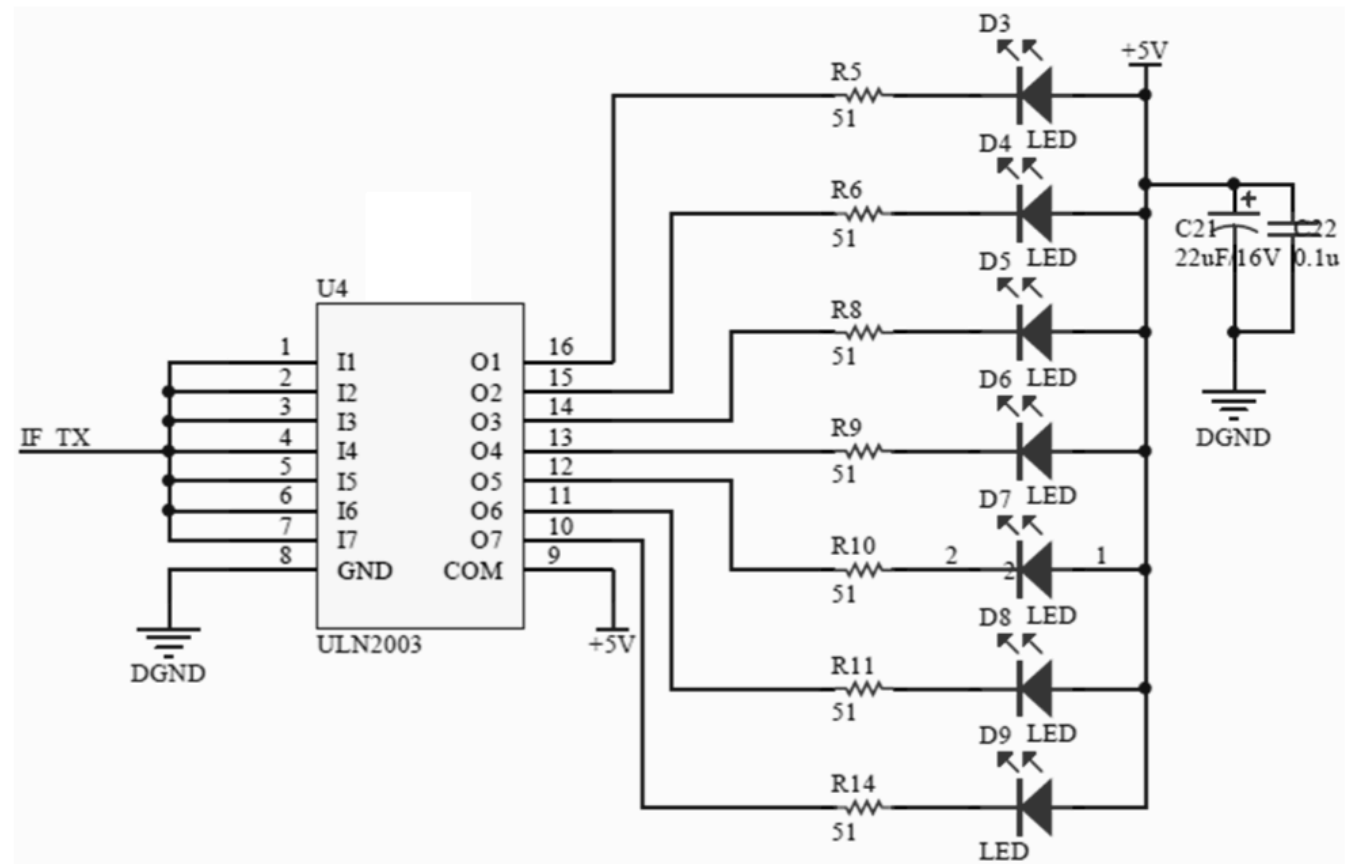

Fig. (10). Circuit diagram of infrared emitter.
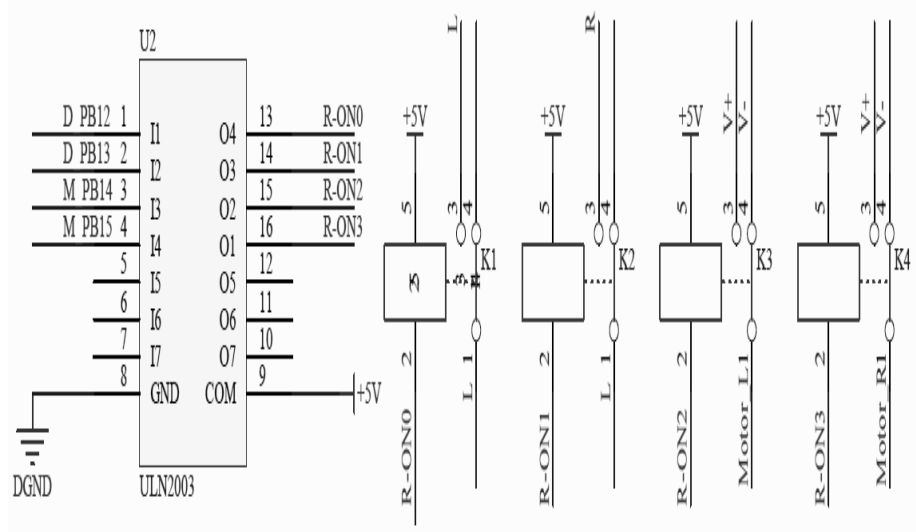

Fig. (11). Circuit diagram of electric curtains.

urgency. In other words, all tasks are grouped into six user task categories and one system task categories. The flowchart of the main control system software is shown in Fig. (12).

\subsection{Programming Design of the ZigBee Coordinator}

The network layer of the ZigBee protocol stack forms a complete ZigBee network mainly. The network layer is mainly responsible for allocating network address to each ZigBee node, sending control commands, receiving the sensing information from the network nodes, and uploading the data received to the STM32F407 microprocessor through the RS232 serial port. The flowchart of ZigBee coordinator program is shown in Fig. (13). The coordinator first completes the initialization of the application layer, then initializes the
CC2530 I/O port and turns on the global interrupt. A ZigBee network is created by initializing a channel. Each network node is authenticated correspondingly before joining the network.

\subsection{ZigBee Network Node Program}

ZigBee network nodes are controlled by the ZigBee coordinator. In the smart home system, the nodes are mainly responsible for collecting sensing information as well as controlling home appliances, electronic control locks and multifunction switch equipment. The flowchart of network nodes is shown in Fig. (14). After the system is powered on, the CC2530 chip initializes the IO port, opens the global interruption, applies for access to the ZigBee network, and sends 


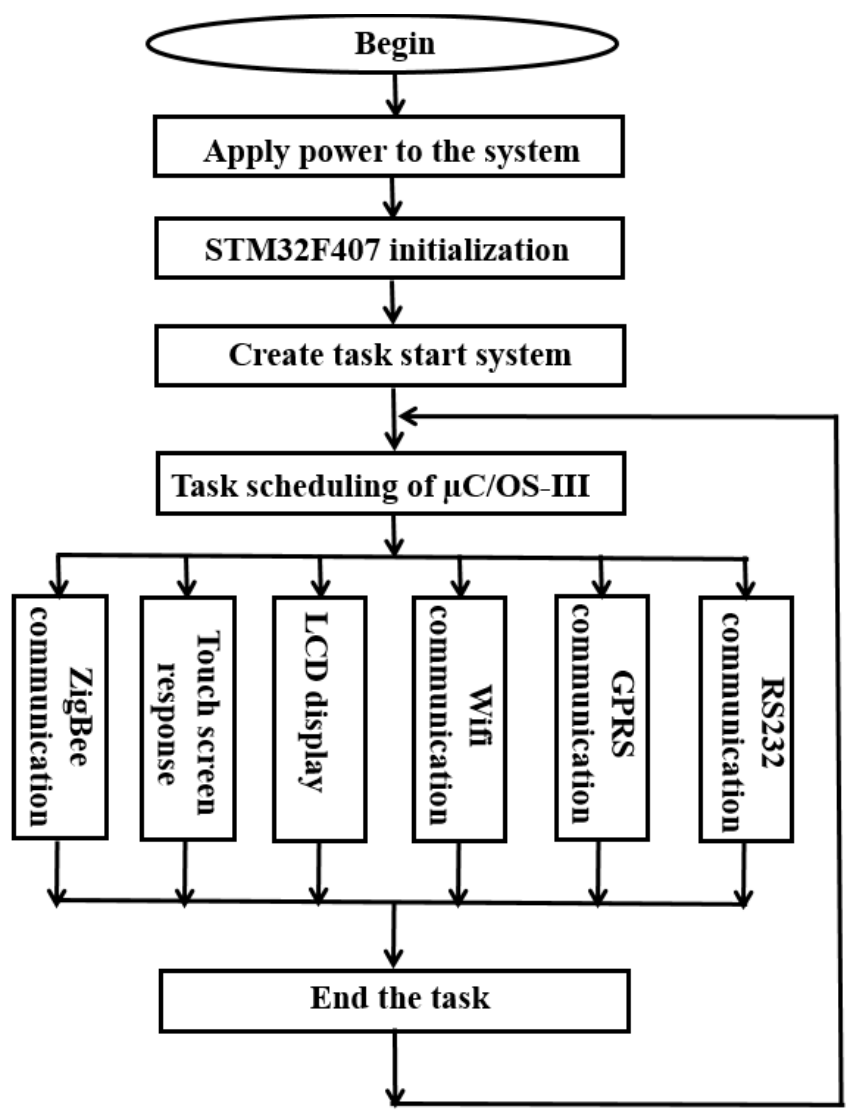

Fig. (12). Flowchart of main control system software.

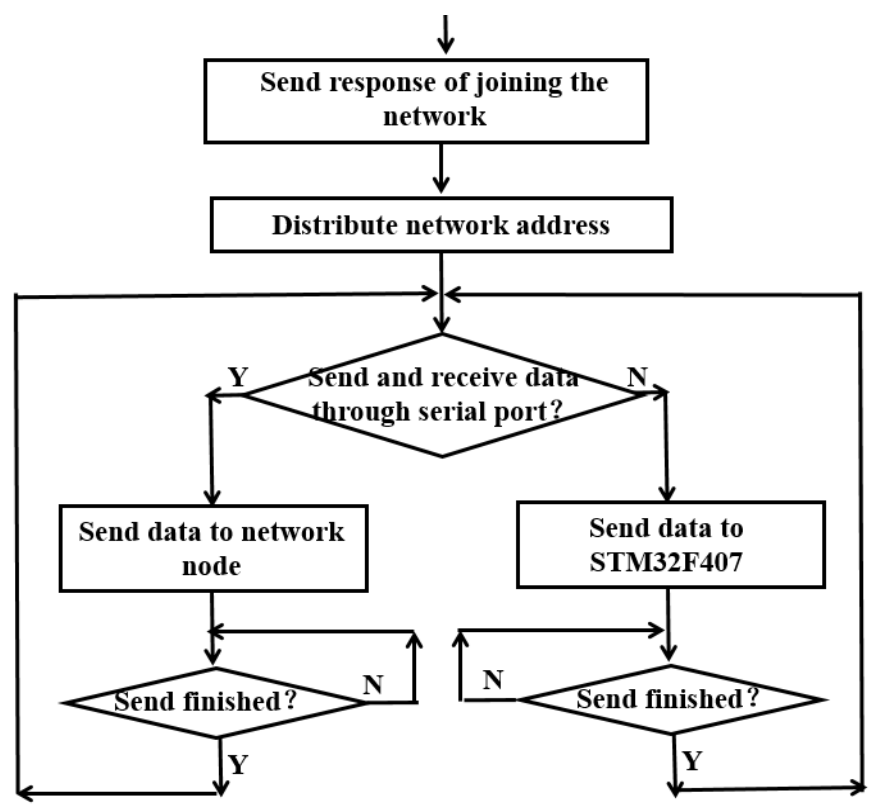

Fig. (13). Flowchart of ZigBee coordinator program. 


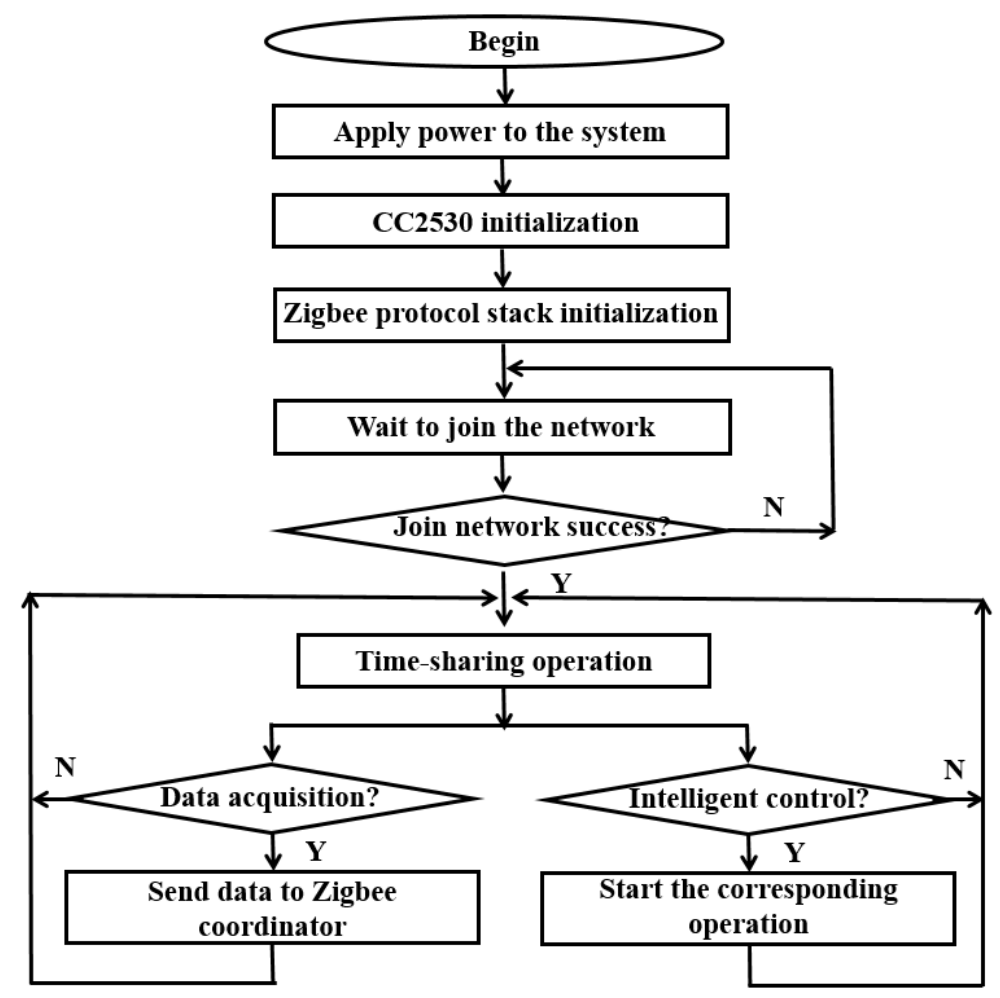

Fig. (14). Flowchart of ZigBee network node program.

the network node registration information to the ZigBee coordinator. Thus, the ZigBee network is formed. By using a time slice polling mechanism, ZigBee network nodes collect sensor information regularly, and upload it to the coordinator. At the same time, ZigBee network nodes receive control commands from the master controller of the smart home system, and start the corresponding operation accordingly.

\subsection{Android App for Smart phones}

Developed based on the open-source Android platform [10], the smart phone app can be run on various Android smart phones and tablets. The smart phone app mainly includes login interface, monitoring setting interface, display and control interfaces. The login interface prompts the user to enter identity information for authentication. Then the authenticated users can enter the monitoring setting interface where the users can select WiFi or GPRS to communicate with the main control system of STM32F407. After a valid connection is established, users can set up the functions of lighting, security, remote control, cameras, etc. Then, users can monitor the tasks running in the smart home system on the display and control interface.

\section{SUMMARY}

STM32F407 microprocessor is used as the master control in this smart home system. With the aided design of ZigBee modules based on CC2530 chip, the system realizes information sharing between various home appliances. Users can operate this system through operations either on the touch screen of STM32F407 master controller on site or on their wireless smart phones or tablets, creating an interconnected
Internet of Things in the home environment. The smart home system has been installed and used in the laboratory. Facts have proved this system to be stable and reliable. This smart home system can be used in various scenarios including home, government office, company, factory floor, which can reduce labor cost effectively.

\section{CONFLICT OF INTEREST}

The authors confirm that this article content has no conflicts of interest.

\section{ACKNOWLEDGEMENTS}

This work is supported by the twelfth five year plan project of Education Science, Guangdong, China (No.2013JK200).

\section{REFERENCES}

[1] M. Chan, D. Estève, C. Escriba, and E. Campo, "A review of smart homes-Present state and future challenges," Computer Methods and Programs in Biomedicine, vol. 91, pp. 55-81, 2008.

[2] R.J. Robles, and T. Kim, "Applications systems and methods in smart home technology: a review," International Journal of Advanced Science and Technology, vol. 15, pp. 37-47, 2010.

[3] Y. Liu, J. Huang, and Z. He, "Design of the automatic jacquard control system based on STM32F407," International Conference on Information Science, Electronics and Electrical Engineering (ISEEE), vol. 2, pp. 1143-1146, 2014.

[4] D.M. Han, and J.H. Lim, "Smart home energy management system using IEEE 802.15.4 and ZigBee. consumer electronics," IEEE Transactions on Consumer Electronics, vol. 56, pp. 1403-1410, 2010. 
[5] A. Wheeler, "Commercial applications of wireless sensor networks using ZigBee," IEEE Communications Magazine, vol.45, pp. 7077, 2007.

[6] K. Lian, S. Hsiao, and W. Sung, "Intelligent multi-sensor control system based on innovative technology integration via ZigBee and Wi-Fi networks," Journal of Network and Computer Applications, vol. 36, pp. 756-767, 2013.

[7] H. Zhang, and W. Kang, "Design of the data acquisition system based on STM32," Procedia Computer Science, vol. 17, pp. 222228, 2013.
[8] J. Tao, and X. Wang, "Low-end video surveillance system implemented with 0V7670 and STM32F407," Microcontrol-lers \& Embedded Systems, vol. 14, pp. 60-63, 2014.

[9] K. Wang, P. Li, J. Liu, and D. Ning, "Application of $\mu \mathrm{c} / \mathrm{os}-\mathrm{II}$ in the design of mine dc electrical prospecting instrument," Procedia Earth and Planetary Science, vol. 3, pp. 485-492, 2011.

[10] R. Chappel, and K. Paliwal, "An educational platform to demonstrate speech processing techniques on Android based smart phones and tablets," Speech Communication, vol. 57, pp. 13-38, 2014.

Received: September 16, 2014

(C) Zhang and Li; Licensee Bentham Open.

This is an open access article licensed under the terms of the Creative Commons Attribution Non-Commercial License (http://creativecommons.org/licenses/by-nc/3.0/) which permits unrestricted, non-commercial use, distribution and reproduction in any medium, provided the work is properly cited. 